\title{
EFEITOS DOS EXERCÍCIOS FÍSICOS NA DENSIDADE MINERAL ÓSSEA DE MULHERES ACIMA DE 50 ANOS: REVISÃO INTEGRATIVA
}

\author{
Jadson Miranda Souza ${ }^{1}$, Maklin Tavares de Almeida ${ }^{1}$, Rodrigo Gomes de Souza Vale ${ }^{2}$, \\ Maria de Nazaré Dias Bello ${ }^{3}$ \& Claudio Joaquim Borba Pinheiro ${ }^{1,4}$
}

\begin{abstract}
RESUMO
SOUZA, J.M.; ALMEIDA, M.T.; VALE, R.G.S.; BELLO, M.N.D.; BORBA-PINHEIRO, C.J. Efeitos dos exercícios físicos na densidade mineral óssea de adultos mais velhos e idosos: revisão integrativa. Perspectivas Online: Biológicas \& Saúde, v. 11, n. 40, p. 11 - 28, 2021.

Objetivo: analisar os dados na literatura do período 2015-2020 sobre os efeitos de distintos exercícios físicos na densidade mineral óssea (DMO) em adultos mais velhos e classifica-los de forma quantitativa. Métodos: trata-se de uma revisão integrativa da literatura, em que na seleção dos artigos, utilizou as plataformas de dados SCIELO, PUBMED, LILACS e BVS. Resultados: foram selecionados quatorze estudos categorizados a seguir: A - treinamento resistido isolado (TRI); Bplataforma vibratória (PV); C- Exercícios multicomponentes (EM). Conclusão:

exercícios físicos podem ter um impacto positivo na DMO, especialmente nas mulheres. Assim, programas de treinamento com mais de um tipo de exercício, usando a PV e também o TRI demonstraram melhores resultados para a DMO no período estudado. Observou-se ainda, que as regiões mais incidentes foram: coluna lombar, colo do fêmur e quadril total. Além disso, não foi possível classificar quantitativamente os métodos de exercícios, devido as diferentes formas metodológicas de abordar os métodos de treinamento.
\end{abstract}

Palavras-chave: Densidade óssea; Exercícios Físicos; Idade avançada.

\footnotetext{
${ }^{1}$ Universidade do Estado do Pará (UEPA). Curso de Educação Física. Laboratório de Treinamento Resistido e Saúde (LERES). Campus XIII, Tucuruí-PA, Brasil

${ }^{2}$ Universidade do Estado do Rio de Janeiro (UERJ). Programa de Pós Graduação Stricto Sensu em Ciências do Esporte. Rio de Janeiro-RJ, Brasil

${ }^{3}$ Secretaria de Estado de Educação SEDUC/PA. Governo do Estado do Pará. Núcleo de Esporte e Lazer (NEL), Belém-PA, Brasil

${ }^{4}$ Instituto Federal de Educação Ciência e Tecnologia do Pará (IFPA) campus de Tucuruí-PA, Brasil

*Claudio Joaquim Borba-Pinheiro. Universidade do Estado do Pará (UEPA), Campus XIII, Tucuruí, Pará, Brasil. Rua Quatro, 20, Bairro: Santa Mônica. CEP: 68455-210 - Tucuruí, Pará, Brasil. Tel.: +55 (94) 98170-0066. e-mail: claudioborba18@gmail.com
} 
Revista Perspectivas Online: Biológicas \& Saúde December/2021, v.11, n. 40 , p. $11-28$ ISSN: 2236-8868 (Online)

DOI: $10.25242 / 8868114020212468$

\title{
EFFECTS OF PHYSICAL EXERCISES ON BONE MINERAL DENSITY OF WOMEN AFTER 50 YEARS: INTEGRATIVE REVIEW
}

\section{Jadson Miranda Souza ${ }^{1}$, Maklin Tavares de Almeida ${ }^{1}$, Rodrigo Gomes de Souza Vale ${ }^{2}$, Maria de Nazaré Dias Bello ${ }^{3} \&$ Claudio Joaquim Borba Pinheiro ${ }^{1,4}$}

\begin{abstract}
SOUZA, J.M.; ALMEIDA, M.T.; VALE, R.G.S.; BELLO, M.N.D.; BORBA-PINHEIRO, C.J. Effects of physical exercises on bone mineral density of women after 50 years: integrative review. Perspectivas Online: Biológicas \& Saúde, v.11 , n. 40 , p. 11- 28, 2021.
\end{abstract}

Objective: to analyze data from studies in the literature for the period 2015-2020 that verified the effects of different physical exercises on BMD in older adult and classify them quantitatively. Methods: it is an integrative literature review, in the articles selection were used the platforms SCIELO, PUBMED, LILACS and BVS. Results: fourteen studies were selected and categorized follows: A - resistance training RT isolated; B- vibration platform (VP); Cmulticomponent exercises (ME). Conclusion: physical exercise can have a positive impact on the BMD in older adults, especially in women. Thus, it was possible to verify that training programs with more than exercise type, using the VP and also the TR, proved to be more effective for BMD in studied period. It was also observed that the most incident regions were: lumbar spine, femoral neck and total hip. In addition, it was not possible to classify quantitatively the exercise methods, due to the different methodological ways of approaching training methods.

Keywords: Bone density; Physical exercise; Older years.

\footnotetext{
${ }^{1}$ University of the State of Pará (UEPA). Physical Education Course. Resistance Training and Health Laboratory (LERES). Campus XIII, Tucuruí-PA, Brazil

${ }^{2}$ State University of Rio de Janeiro (UERJ). Stricto Sensu Graduate Program in Sports Science. Rio de Janeiro-RJ, Brazil

${ }^{3}$ Federal Institute of Education, Science and Technology of Pará (IFPA) Tucuruí-PA campus, Brazil* Claudio Joaquim BorbaPinheiro. State University of Pará (NEL), Belém-PA, Brazil

${ }^{4}$ Instituto Federal de Educação Ciência e Tecnologia do Pará (IFPA) campus de Tucuruí-PA, Brazil

*Claudio Joaquim Borba-Pinheiro. Universidade do Estado do Pará (UEPA), Campus XIII, Tucuruí, Pará, Brazil. Street Quatro, 20, District: Santa Mônica. Postal Code: 68455-210 - Tucuruí, Pará, Brazil. Phone.: +55 (94) 98170-0066. e-mail: claudioborba18@gmail.com
} 


\section{INTRODUÇÃO}

O aumento na expectativa de vida mundial tornou-se um fator singular para a sociedade, pois primeira vez na história, grande parte da população apresenta uma estimativa acima dos 60 anos. Para 2050, estima-se que, indivíduos com 60 anos ou mais atinjam os 2 bilhões, superando os 900 milhões de 2015, além disso, 125 milhões de indivíduos possuirão 80 anos ou mais no mundo (OPAS, 2018).

O número de pessoas com mais de 60 anos ultrapassou os 30 milhões em 2017, o sexo feminino ocupa a maior parte desse número, 16,9 milhões (56\%), enquanto que os idosos do sexo masculino equivalem a 13,3 milhões (44\%), além disso, de acordo com o Instituto Brasileiro de Geografia e Estatística (IBGE), em 2031, o número de idosos vai superar o de crianças e adolescentes de até 14 anos (BRASIL, 2018).

Se por um lado isto é positivo, pois mostra o aumento da taxa de longevidade, por outro torna-se uma questão preocupante, já que o envelhecimento é uma consequência do impacto constante de várias lesões celulares ao longo dos anos. Ele tem como principal consequência a diminuição gradual das funções físicas e psíquicas, além do risco progressivo de patologias e da morte. Não obstante, tais alterações não são uma regra, tendo em vista que é possível a saúde com preservação funcional em pessoas com 70 anos e outras, na mesma idade, com fragilidade e dependência física/funcional, onde a diferença está na manutenção das variáveis relacionadas aos hábitos de vida saudável (CHAMOSCHINE et al., 2018; MACNEE et al., 2014; MANHÃES et al., 2018).

Diante disso, verifica-se que boa parte dos idosos perde sua independência quando há a ocorrência de quedas, que são consequências potencial do baixo nível de atividade física, o que afeta também os familiares, cuidadores e/ou indivíduos próximos, como também os âmbitos biopsicossociais dessas vítimas, com possibilidades aumentadas de internações e ainda sujeitos a desenvolvimento de complicações hospitalares (ALMEIDA et al., 2019).

É claro que há avanços na atenção básica de saúde, com políticas públicas, melhores condições sanitárias e da medicina com as avalições por imagem, que ajudam a melhorar as condições de vida. Há, também, uma relação entre a evolução da tecnologia e o aumento da expectativa de vida, considerando distintos campos beneficiados pelos estudos tecnocientíficos, que incluem novos métodos para prevenção, diagnósticos, tratamentos, medicamentos, educação e comunicação em saúde (LEITE; ROSA, 2017).

Entretanto, a tecnologia que hora ajuda na saúde, também pode prejudicar, como é o caso da execução de tarefas que se tornou simplificada, diminuindo a duração e a intensidade das atividades físicas e consequentemente, favoreceu a exposição aos comportamentos sedentários. Desse modo, tais alterações nos estilos de vida, por este ponto de vista, podem provocar resultados negativos à saúde (ACSM, 2014; CHAMOSCHINE et al., 2018).

Outrossim, no que se refere a densidade mineral óssea (DMO), ela é determinada por fases ativas de remodelação, a qual é caracterizada pela reabsorção e produção de tecido ósseo. O envelhecimento está relacionado as alterações entre os processos de remodelação, tendo como consequência a gradual diminuição na DMO e a perda progressiva da massa óssea, o que resulta em fragilidade da estrutura e da resistência física do osso e favorece a formação do quadro clínico de osteopenia e/ou osteoporose (VELHO, 2014).

As ocorrências da DMO, geralmente, se dão no sexo feminino, na pré-menopausa e, com maior frequência, na pós-menopausa (VELHO, 2014). Assim, o ato de se exercitar 
regularmente ajuda na promoção de um envelhecimento saudável, além do controle da DMO (BARROS; BELMONTE, 2016).

A atividade física com impacto promove microfraturas no esqueleto, onde o exercício realiza pressão sob células chamadas osteócito e consequentemente a atividade osteoblástica, estimulando o metabolismo da remodelação óssea (DISSAT, 2009). Neste sentido, a literatura cientifica traz vários estudos que incluem métodos de exercícios físicos com efeitos sobre a DMO, entre estes: treinamento resistido, hidroginástica, combinado com dois ou mais exercícios (CÂMARA; BASTOS; VOLPE, 2012; ACSM, 2014; BORBA-PINHEIRO et al., 2016).

O treinamento resistido (TR) isolado ou associado se mostra eficiente ao gerar estímulos tanto na massa muscular, quanto óssea, auxiliando na diminuição da perda de DMO (JESUS et al., 2019). O American College of Sports Medicine (ACSM) recomenda para indivíduos que possuem baixa DMO, a realização de exercícios aeróbicos, podendo ser combinado com resistidos. A indicação usual, para pessoas com perda de DMO, é prescrever exercícios com uma intensidade moderada que não gere ou intensifique dores (ACSM, 2014).

Os estudos de revisão sistemática e metanálise sobre exercício e DMO são apresentados na literatura científica e mostram que podem ser eficientes como estratégia não medicamentosa para manutenção da DMO associados a outras variáveis de risco de quedas (SANTOS; BORGES, 2010), porém ainda existem lacunas sobre novas necessidades como, os métodos de exercícios que podem ser mais efetivos para DMO. Dessa forma, buscou-se com o presente estudo, diminuir as possíveis lacunas relacionadas à aplicação de métodos de exercícios físicos para prevenção e tratamento da perda da DMO. Neste sentido, surgiu o seguinte problema da pesquisa: Quais os tipos de exercícios físicos que são mais eficientes para o aumento na DMO de adultos mais velhas?

Diante do exposto, o objetivo deste estudo foi analisar os estudos na literatura científica do período 2015-2020 que verificam os efeitos em distintos tipos de exercícios físicos na DMO de adultos mais velhos, e classifica-los de forma quantitativa.

\section{METODOLOGIA}

Trata-se de um estudo com características de revisão bibliográfica do tipo integrativa, que consiste na análise de pesquisas relevantes para o campo prático de atuação, facilitando a ênfase de pontos positivos, negativos, evolução e probabilidades sobre determinada temática, por meio de seis etapas para sua elaboração (MENDES; SILVEIRA; GALVÃO, 2008).

Para o desenvolvimento da pesquisa, serão utilizadas 6 etapas, as quais são: 1- criação da pergunta-chave; 2- busca na literatura; 3 - coleta de informações; 4- avaliação criteriosa dos artigos incluídos; 5- discussão dos resultados e 6- apresentação da revisão integrativa (GRUPO ANIMA EDUCAÇÃO, 2014).

Ademais, o presente estudo está de baseado nas diretrizes do PRISMA (Preferred Reporting Items for Systematic Reviews and Meta-analyses), que possui como objetivo, auxiliar os autores a desenvolverem de maneira mais eficiente as pesquisas bibliográficas, por meio de um checklist com 27 tópicos (GALVÃO; PANSANI; HARRAD, 2015).

Persp. Online: biol \& saúde, Campos dos Goytacazes, 40 (11) 11-28, 2021 
Para a seleção dos artigos utilizou-se as plataformas de bancos de dados SCIELO (Scientific Eletronic Library Online), PUBMED (National Library of Medicine), LILACS (Literatura Latino-Americana e do Caribe em Ciências da Saúde) e BVS (Biblioteca Virtual em Saúde Brasil). Os descritores que foram usados nas buscas estavam englobados como parte do vocabulário nos bancos de indexadores DeCs (Descritores em ciências da Saúde) e MeSH (Medical Subject Heading), os quais são: "bone density", "physical exercise", "osteoporosis", "densidad ósea", "ejercicio físico", "osteoporosis", "densidade óssea", "exercício físico", "osteoporose". A articulação desses descritores se deu pares, pela aplicação dos operadores booleanos "AND/OR/", nas línguas portuguesa, inglesa e espanhola.

Os critérios de inclusão elencados foram: artigos revisão sistemática e/ou meta análise, ensaios clínicos randomizados, publicados na íntegra, de acesso público e online, nas línguas portuguesa, inglesa ou espanhola, que tinham como amostra indivíduos onde a média de idade foi igual ou superior a 50 anos, e publicados entre os anos de 2015 e 2020.

Para exclusão, foram desconsiderados as monografias, dissertações, teses, relatos de experiência, estudos de caso, editoriais, produções científicas que mostravam somente o resumo e delimitavam uma faixa-etária menor que 50 anos. Descartou-se também artigos que não tratassem essencialmente do tema no período proposto e pesquisas duplicadas, mantendo somente a primeira versão detectada.

\section{RESULTADOS}

A busca executada nos bancos de dados, resultou em um total de 20.617 artigos, distribuídos nas plataformas: PUBMED, SCIELO, LILACS e BVS/MS, conforme apresentado na Figura 1. Posteriormente houve o descarte de 16 estudos duplicados e 20.563 pesquisas excluídas por não se adequarem aos critérios de seleção, totalizando 38 estudos préselecionados, destas, 15 documentos foram descartados por não se adequarem a temática proposta.

Por conseguinte, foram avaliados 23 documentos na íntegra, sendo excluídos 9 trabalhos por não satisfazerem os objetivos da presente pesquisa, como demonstra a Figura 1. Os estudos foram selecionados por dois pesquisadores e revisados por um terceiro. 


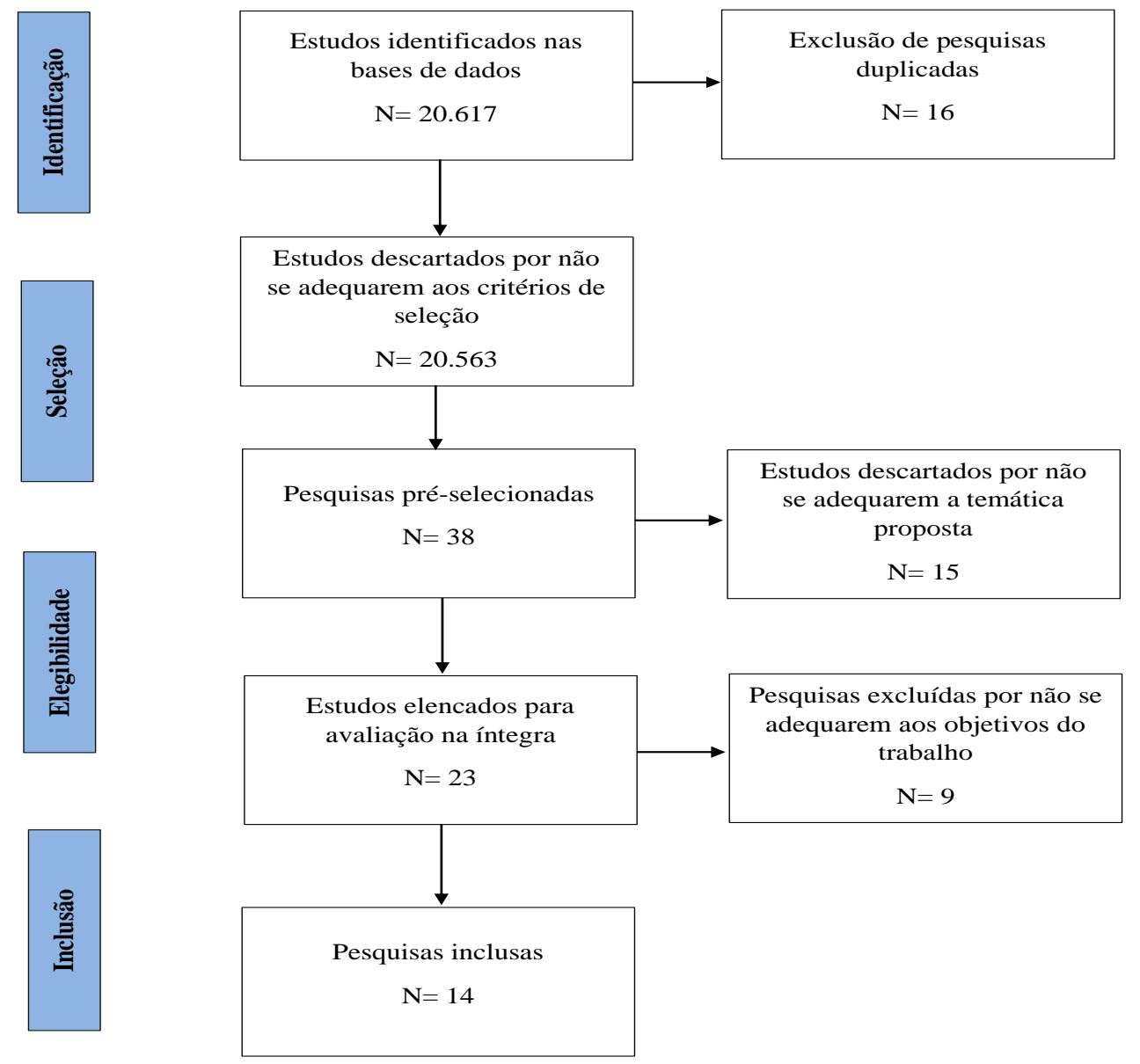

Figura 1 - Fluxograma da pesquisa de revisão integrativa, baseada nos moldes do Prisma.

Após os procedimentos metodológicos de inclusão e exclusão, tem-se como resultados quatorze pesquisas, apresentadas no Quadro 1, as quais foram extraídas por um formulário elaborado pelos autores, contendo as informações consideradas específicas e que melhor resumem os trabalhos. 


\begin{tabular}{|c|c|c|c|c|c|c|c|}
\hline \multicolumn{8}{|c|}{ Quadro 1. Apresenta os resultados para a seleção de estudos de acordo com os critérios estabelecidos } \\
\hline $\mathbf{N}^{\mathbf{0}}$ & AUTORES & TÍTULO & OBJETIVO & $\begin{array}{c}\text { TIPO DE } \\
\text { EXERCÍCIO }\end{array}$ & MÉTODOS & RESULTADOS & CONCLUSÃO \\
\hline 1 & $\begin{array}{l}\text { Kemmler et } \\
\text { al. }(2020)\end{array}$ & $\begin{array}{l}\text { Effects of Different } \\
\text { Types of Exercise on } \\
\text { Bone Mineral } \\
\text { Density in } \\
\text { Postmenopausal } \\
\text { Women A Systematic } \\
\text { Review and Meta- } \\
\text { analysis. }\end{array}$ & $\begin{array}{l}\text { Determinar o efeito } \\
\text { de diferentes tipos de } \\
\text { exercício na DMO de } \\
\text { algumas áreas em } \\
\text { mulheres na pós- } \\
\text { menopausa. }\end{array}$ & $\begin{array}{c}\text { Exercício TR. } \\
\text { Exercício de } \\
\text { sustentação de peso } \\
\text { corporal. } \\
\text { Exercício de } \\
\text { sustentação de peso } \\
\text { combinado com } \\
\text { exercício de } \\
\text { treinamento resistido. }\end{array}$ & $\begin{array}{l}\text { Revisão } \\
\text { sistemática da } \\
\text { literatura. }\end{array}$ & $\begin{array}{l}\text { Efeitos positivos com diferentes } \\
\text { tipos de exercícios na DMO da } \\
\text { coluna lombar, colo do fêmur e } \\
\text { quadril. } \\
\text { Com relação aos } 3 \text { métodos de } \\
\text { exercícios abordados na DMO da } \\
\text { coluna lombar, colo do fêmur e } \\
\text { quadril, não foram observadas } \\
\text { diferenças significativas entre eles. }\end{array}$ & $\begin{array}{c}\text { Demonstrou evidências } \\
\text { adicionais para o efeito } \\
\text { favorável do exercício } \\
\text { na DMO, independente } \\
\text { do tipo de exercício. } \\
\text { Contudo, para gerar } \\
\text { protocolos de exercícios } \\
\text { padronizados, as meta- } \\
\text { análises podem ser uma } \\
\text { ferramenta muito } \\
\text { imprecisa. }\end{array}$ \\
\hline 2 & $\begin{array}{l}\text { Shojaa et al. } \\
\quad(2020 \mathrm{a})\end{array}$ & $\begin{array}{c}\text { Effects of dynamic } \\
\text { resistance exercise on } \\
\text { bone mineral density } \\
\text { in postmenopausal } \\
\text { women: a systematic } \\
\text { review and meta- } \\
\text { analysis with special } \\
\text { emphasis on exercise } \\
\text { parameters. }\end{array}$ & $\begin{array}{l}\text { Determinar o efeito } \\
\text { do treinamento } \\
\text { resistido isolado na } \\
\text { DMO da coluna } \\
\text { lombar e fêmur em } \\
\text { mulheres na pós- } \\
\text { menopausa, } \\
\text { comparadas com } \\
\text { grupos de controle. }\end{array}$ & $\begin{array}{c}\text { Treinamento resistido } \\
\text { (TR). }\end{array}$ & $\begin{array}{c}\text { Revisão } \\
\text { sistemática e } \\
\text { meta-análise. }\end{array}$ & $\begin{array}{l}\text { Moderadores dos efeitos do } \\
\text { exercício, isto é, "duração", "tipo", } \\
\text { "frequência", "intensidade", foram } \\
\text { utilizados para analisar os } \\
\text { subgrupos estabelecidos. } \\
\text { Para alterações da DMO no fêmur, } \\
\text { não houve diferenças entre as } \\
\text { categorias de moderadores. } \\
\text { Na baixa frequência de } \\
\text { treinamento, verificou-se alterações } \\
\text { da DMO significativamente na } \\
\text { coluna lombar e quadril total, } \\
\text { comparados a alta frequência } \\
\text { O exercício com pesos livres foi } \\
\text { significativamente superior aos de } \\
\text { TR na DMO quadril total. }\end{array}$ & $\begin{array}{c}\text { As análises não } \\
\text { permitiram derivar } \\
\text { recomendações de } \\
\text { exercícios significativas, } \\
\text { já que o estudo observou } \\
\text { um efeito baixo a } \\
\text { moderado considerável } \\
\text { do treinamento resistido } \\
\text { DMO no público-alvo. }\end{array}$ \\
\hline 3 & $\begin{array}{l}\text { Mainer; } \\
\text { Morales; } \\
\text { Sagarra } \\
(2019) \\
\end{array}$ & $\begin{array}{c}\text { Efectos de las } \\
\text { plataformas } \\
\text { vibratorias en la salud }\end{array}$ & $\begin{array}{l}\text { Conhecer os últimos } \\
\text { avanços nos } \\
\text { treinamentos em } \\
\text { plataformas } \\
\end{array}$ & $\begin{array}{c}\text { Exercícios na } \\
\text { plataforma vibratória. }\end{array}$ & $\begin{array}{l}\text { Revisões } \\
\text { sistemáticas e } \\
\text { meta-análises. }\end{array}$ & $\begin{array}{c}\text { Há maior conformidade do } \\
\text { treinamento com a } \\
\text { equipamento/marca Power Plate } \\
\text { comparado ao Galileo, mas o }\end{array}$ & $\begin{array}{c}\text { Os benefícios e } \\
\text { malefícios da PV em } \\
\text { mulheres na pós- } \\
\text { menopausa ainda não }\end{array}$ \\
\hline
\end{tabular}

Persp. Online: biol \& saúde, Campos dos Goytacazes, 40 (11) 11-28, 2021

ojs3.perspectivasonline.com.br 


\begin{tabular}{|c|c|c|c|c|c|c|c|}
\hline & & $\begin{array}{l}\text { ósea en mujeres } \\
\text { posmenopáusicas. }\end{array}$ & $\begin{array}{l}\text { vibratórias para a } \\
\text { melhoria da massa } \\
\text { óssea em mulheres na } \\
\text { pós-menopausa. }\end{array}$ & & & $\begin{array}{c}\text { primeiro é usado em menos } \\
\text { tentativas do que o último e ao } \\
\text { compará-lo com outro treinamento, } \\
\text { não foram obtidas diferenças } \\
\text { significativas, talvez porque o } \\
\text { movimento oscilatório é mais } \\
\text { benéfico para essa população do } \\
\text { que o movimento triaxial. }\end{array}$ & $\begin{array}{l}\text { são explicitamente } \\
\text { conhecidos e, portanto, } \\
\text { seria interessante } \\
\text { realizar mais ensaios } \\
\text { clínicos, alternando com } \\
\text { outros treinamentos para } \\
\text { entender completamente } \\
\text { o papel desta terapia. } \\
\end{array}$ \\
\hline 4 & $\begin{array}{l}\text { Shojaa et al. } \\
\text { (2020b) }\end{array}$ & $\begin{array}{l}\text { Effect of Exercise } \\
\text { Training on Bone } \\
\text { Mineral Density in } \\
\text { Post-menopausal } \\
\text { Women: A } \\
\text { Systematic Review } \\
\text { and Meta-Analysis of } \\
\text { Intervention Studies }\end{array}$ & $\begin{array}{l}\text { Resumir os efeitos } \\
\text { dos exercícios físicos } \\
\text { na DMO de mulheres } \\
\text { que já passaram da } \\
\text { menopausa. }\end{array}$ & $\begin{array}{l}\text { Aeróbicos. } \\
\begin{array}{l}\text { Sustentação de peso } \\
\text { corporal. }\end{array} \\
\text { Treinamento } \\
\text { resistido. } \\
\text { Saltos. } \\
\text { Tai Chi. }\end{array}$ & $\begin{array}{l}\text { Revisões } \\
\text { sistemáticas e } \\
\text { meta-análises. }\end{array}$ & $\begin{array}{l}\text { Para coluna lombar, os melhores } \\
\text { resultados foram a combinação de } \\
\text { saltos com TR e exercícios com o } \\
\text { peso do corpo. } \\
\text { Quando verificado o pescoço do } \\
\text { fêmur, os exercícios que mostraram } \\
\text { os maiores resultados foram os que } \\
\text { os indivíduos não tinham que } \\
\text { sustentar o peso do corpo, como por } \\
\text { exemplo o ciclismo. } \\
\text { Para o quadril total, os maiores } \\
\text { resultados foram os que } \\
\text { combinavam saltos com TR e } \\
\text { exercícios com o peso do corpo. }\end{array}$ & $\begin{array}{l}\text { O estudo falhou em } \\
\text { definir um protocolo de } \\
\text { treinamento mais efetivo } \\
\text { para o aumento da massa } \\
\text { óssea nas regiões de } \\
\text { interesse, isso se deve a } \\
\text { grande heterogeneidade } \\
\text { e grande quantidade de } \\
\text { exercícios verificados } \\
\text { nos estudos. }\end{array}$ \\
\hline 5 & $\begin{array}{l}\text { Simas et al. } \\
\text { (2017) }\end{array}$ & $\begin{array}{l}\text { Effects of water- } \\
\text { based exercise on } \\
\text { bone health of } \\
\text { middle-aged and } \\
\text { older adults: a } \\
\text { systematic review } \\
\text { and meta-analysis }\end{array}$ & $\begin{array}{l}\text { Verificar se } \\
\text { exercícios em meio } \\
\text { aquático são eficazes } \\
\text { na prevenção } \\
\text { relacionada à idade } \\
\text { deterioração óssea em } \\
\text { adultos de meia-idade } \\
\text { e idosos. }\end{array}$ & $\begin{array}{l}\text { Exercícios } \\
\text { executados em meio } \\
\text { aquático } \\
\text { (hidroginástica/ } \\
\text { natação). } \\
\text { Exercícios em terra } \\
\text { (treinamento } \\
\text { resistido/ de força). }\end{array}$ & $\begin{array}{l}\text { Revisões } \\
\text { sistemáticas e } \\
\text { meta-análises. }\end{array}$ & $\begin{array}{l}\text { Efeitos significativos na lombar e } \\
\text { colo femoral foram favoráveis aos } \\
\text { exercícios aquáticos comparados } \\
\text { aos controles. } \\
\text { Porém, o grupo de exercícios } \\
\text { resistido/força, a favor do segundo } \\
\text { foi ( } \mathrm{p}<0,05) \text { melhor na DMO da } \\
\text { lombar comparado ao aquático. } \\
\text { Não houveram diferenças entre os } \\
\text { grupos no colo do fêmur. }\end{array}$ & $\begin{array}{l}\text { Exercícios em meio } \\
\text { aquático podem } \\
\text { promover benefícios a } \\
\text { respeito da manutenção } \\
\text { e melhoria da saúde } \\
\text { óssea em mulheres na } \\
\text { pós menopausa, no } \\
\text { entanto, eles se } \\
\text { mostraram menos } \\
\text { eficientes quando } \\
\text { comparados a exercícios } \\
\text { feitos em terra. }\end{array}$ \\
\hline 6 & $\begin{array}{l}\text { Kim; Moon; } \\
\text { Jin (2016) }\end{array}$ & $\begin{array}{l}\text { The effects of } \\
\text { exercise training and } \\
\text { type of exercise }\end{array}$ & $\begin{array}{l}\text { Avaliar os efeitos do } \\
\text { treinamento físico e o } \\
\text { tipo de treinamento }\end{array}$ & $\begin{array}{c}\text { Treinamento } \\
\text { aeróbico. }\end{array}$ & $\begin{array}{l}\text { Revisões } \\
\text { sistemáticas e } \\
\text { meta-análises. }\end{array}$ & $\begin{array}{l}\text { A combinação (aeróbicos e TR) } \\
\text { mostraram melhorias na DMO da } \\
\text { coluna lombar, pescoço do fêmur e }\end{array}$ & $\begin{array}{l}\text { Exercícios físicos podem } \\
\text { melhorar a DMO nas } \\
\text { regiões de interesse }\end{array}$ \\
\hline
\end{tabular}

Persp. Online: biol \& saúde, Campos dos Goytacazes, 40 (11) 11-28, 2021

ojs3.perspectivasonline.com.br 


\begin{tabular}{|c|c|c|c|c|c|c|c|}
\hline & & $\begin{array}{l}\text { training on changes } \\
\text { in bone mineral } \\
\text { denstiy in Korean } \\
\text { postmenopausal } \\
\text { women: a systematic } \\
\text { review }\end{array}$ & $\begin{array}{l}\text { físico sobre as } \\
\text { mudanças na DMO } \\
\text { em mulheres pós- } \\
\text { menopáusicas } \\
\text { coreanas. }\end{array}$ & $\begin{array}{l}\text { TR. } \\
\text { Treinamento misto } \\
\text { (Aeróbico/TR). }\end{array}$ & & $\begin{array}{c}\text { trocânter, no entanto os } \\
\text { treinamentos separados não } \\
\text { mostraram resultados consistentes. }\end{array}$ & $\begin{array}{l}\text { verificadas, em mulheres } \\
\text { na pós menopausa. O } \\
\text { tipo de exercício pode } \\
\text { ser crucial para melhoria } \\
\text { da saúde óssea dessa } \\
\text { população. }\end{array}$ \\
\hline 7 & $\begin{array}{l}\text { Fratini; } \\
\text { Bonci; Bull } \\
\quad(2016)\end{array}$ & $\begin{array}{c}\text { Whole Body } \\
\text { Vibration Treatments } \\
\text { in Postmenopausal } \\
\text { Women Can Improve } \\
\text { Bone Mineral } \\
\text { Density: Results of a } \\
\text { Stimulus Focussed } \\
\text { Meta-Analysis }\end{array}$ & $\begin{array}{c}\text { Revisar } \\
\text { sistematicamente a } \\
\text { literatura para avaliar } \\
\text { o efeito dos } \\
\text { tratamentos de } \\
\text { vibração de corpo } \\
\text { inteiro sobre a DMO } \\
\text { em mulheres na pós- } \\
\text { menopausa. }\end{array}$ & $\begin{array}{l}\text { Exercícios em } \\
\text { plataformas } \\
\text { vibratórias. }\end{array}$ & $\begin{array}{l}\text { Revisões } \\
\text { sistemáticas e } \\
\text { meta-análises. }\end{array}$ & $\begin{array}{l}\text { Na região do quadril e lombar } \\
\text { houve uma mudança significativa } \\
\text { na DMO para as sessões de } \\
\text { exercícios vibratórios na postura } \\
\text { estática. }\end{array}$ & $\begin{array}{l}\text { Exercícios com vibração } \\
\text { do corpo melhorou a } \\
\text { DMO do quadril e } \\
\text { lombar em mulheres na } \\
\text { pós menopausa. No } \\
\text { entanto, os protocolos } \\
\text { precisam de analises } \\
\text { mais aprofundadas. }\end{array}$ \\
\hline 8 & $\begin{array}{c}\text { Qiu; Ma; } \\
\text { Jiang (2020) }\end{array}$ & $\begin{array}{l}\text { Isoflavone combined } \\
\text { with exercise on bone } \\
\text { mineral density in } \\
\text { postmenopausal } \\
\text { women: A systematic } \\
\text { review and meta- } \\
\text { analysis of } \\
\text { randomized } \\
\text { controlled trials }\end{array}$ & $\begin{array}{l}\text { Avaliar os efeitos da } \\
\text { suplementação de } \\
\text { isoflavonas } \\
\text { combinada com o } \\
\text { treinamento físico na } \\
\text { (DMO) em mulheres } \\
\text { na pós-menopausa. }\end{array}$ & $\begin{array}{c}\text { Exercícios aeróbicos. } \\
\text { TR. } \\
\text { Treinamento de } \\
\text { força. } \\
\text { Caminhada. }\end{array}$ & $\begin{array}{l}\text { Revisão } \\
\text { sistemática e } \\
\text { meta-análise. }\end{array}$ & $\begin{array}{l}\text { Os exercícios combinados com a } \\
\text { suplementação de isoflavona, não } \\
\text { mostraram melhorias na DMO. A } \\
\text { intervenção combinada não } \\
\text { mostrou efeitos aditivos na DMO } \\
\text { em comparação com qualquer uma } \\
\text { das intervenções sozinha. }\end{array}$ & $\begin{array}{l}\text { As intervenções com } \\
\text { isoflavona e treinamento } \\
\text { físico não tiveram } \\
\text { efeitos estatísticos na } \\
\text { perda de DMO. No } \\
\text { entanto, ensaios clínicos } \\
\text { bem planejados com } \\
\text { isoflavona de soja de } \\
\text { longo prazo e exercícios } \\
\text { podem ser melhores na } \\
\text { DMO. }\end{array}$ \\
\hline 9 & $\begin{array}{l}\text { Dionello et } \\
\text { al. (2016) }\end{array}$ & $\begin{array}{c}\text { Effects of whole } \\
\text { body vibration } \\
\text { exercises on bone } \\
\text { mineral density of } \\
\text { women with } \\
\text { postmenopausal } \\
\text { osteoporosis without } \\
\text { medications novel } \\
\text { findings and literature } \\
\text { review } \\
\end{array}$ & $\begin{array}{l}\text { Revisar a literatura } \\
\text { sobre o efeito do } \\
\text { exercício de vibração } \\
\text { de corpo inteiro na } \\
\text { DMO em pacientes } \\
\text { com osteoporose pós- } \\
\text { menopausa sem } \\
\text { medicamentos. }\end{array}$ & $\begin{array}{l}\text { Exercícios em } \\
\text { plataformas } \\
\text { vibratórias. }\end{array}$ & $\begin{array}{c}\text { Revisão } \\
\text { sistemática. }\end{array}$ & $\begin{array}{l}\text { Dos } 12 \text { artigos, } 7 \text { demonstraram } \\
\text { melhora da DMO favoráveis aos } \\
\text { exercícios de vibração de corpo } \\
\text { inteiro não associados a } \\
\text { medicamentos. }\end{array}$ & $\begin{array}{c}\text { Os exercícios em PV são } \\
\text { opções não } \\
\text { farmacológicas } \\
\text { relevantes, sendo } \\
\text { recomendadas para o } \\
\text { tratamento da } \\
\text { osteoporose pós- } \\
\text { menopausa. }\end{array}$ \\
\hline
\end{tabular}

Persp. Online: biol \& saúde, Campos dos Goytacazes, 40 (11) 11-28, 2021

ojs3.perspectivasonline.com.br 


\begin{tabular}{|c|c|c|c|c|c|c|c|}
\hline 10 & $\begin{array}{l}\text { Cascales; } \\
\text { Arias; } \\
\text { Alcaraz, } \\
\text { (2019) }\end{array}$ & $\begin{array}{c}\text { Effects of Two } \\
\text { Different } \\
\text { Neuromuscular } \\
\text { Training Protocols on } \\
\text { Regional Bone Mass } \\
\text { in Postmenopausal } \\
\text { Women: A } \\
\text { Randomized } \\
\text { Controlled Trial } \\
\end{array}$ & $\begin{array}{l}\text { Analisar o efeito de } \\
24 \text { semanas de } \\
\text { vibração de corpo } \\
\text { inteiro e treinamento } \\
\text { multicomponente na } \\
\text { massa óssea da } \\
\text { coluna lombar e do } \\
\text { colo do fêmur. }\end{array}$ & $\begin{array}{c}\text { Exercícios em } \\
\text { plataformas } \\
\text { vibratórias. } \\
\text { Drop jump. } \\
\text { Atividade aeróbica. }\end{array}$ & $\begin{array}{l}\text { Ensaio clínico } \\
\text { randomizado. }\end{array}$ & $\begin{array}{l}\text { Houve aumentos significativos na } \\
\text { massa óssea da coluna lombar, com } \\
\text { as plataformas vibratórias. Os } \\
\text { treinamentos com } \\
\text { multicomponentes mostraram } \\
\text { alterações importantes no conteúdo } \\
\text { mineral ósseo da coluna lombar. No } \\
\text { colo femoral, não houve aumento } \\
\text { estatístico. } \\
\end{array}$ & $\begin{array}{c}\text { Os exercícios em } \\
\text { plataformas vibratórias e } \\
\text { multicomponentes, } \\
\text { podem neutralizar a } \\
\text { rápida perda da DMO de } \\
\text { mulheres na pós- } \\
\text { menopausa. }\end{array}$ \\
\hline 11 & $\begin{array}{l}\text { Khan et al., } \\
\text { (2019) }\end{array}$ & $\begin{array}{c}\text { Effects of } \\
\text { osteoanabolic } \\
\text { exercises on bone } \\
\text { mineral density of } \\
\text { osteoporotic females: } \\
\text { A randomized } \\
\text { controlled trial }\end{array}$ & $\begin{array}{l}\text { Identificar o impacto } \\
\text { de diferentes regimes } \\
\text { de exercícios na } \\
\text { melhoria da DMO em } \\
\text { mulheres com } \\
\text { osteoporose. }\end{array}$ & $\begin{array}{l}\text { Aeróbico. } \\
\text { Anaeróbico. } \\
\text { Osteoanabólico } \\
\text { (Aeróbico + } \\
\text { Anaeróbico). }\end{array}$ & $\begin{array}{l}\text { Ensaio clínico } \\
\text { randomizado. }\end{array}$ & $\begin{array}{c}\text { A análise post hoc mostra que os } \\
\text { exercícios osteoanabólicos foram } \\
\text { mais significativos em comparação } \\
\text { com os exercícios aeróbios e } \\
\text { anaeróbio. }\end{array}$ & $\begin{array}{c}\text { Os exercícios } \\
\text { apresentam melhora para } \\
\text { osteoporose; entretanto, } \\
\text { o osteoanabólico } \\
\text { aumentou } \\
\text { significativamente a } \\
\text { DMO. }\end{array}$ \\
\hline 12 & $\begin{array}{l}\text { Oliveira et } \\
\text { al., (2019) }\end{array}$ & $\begin{array}{l}\text { Effects of Whole- } \\
\text { Body Vibration } \\
\text { Versus Pilates } \\
\text { Exercise on Bone } \\
\text { Mineral Density in } \\
\text { Postmenopausal } \\
\text { Women: A } \\
\text { Randomized and } \\
\text { Controlled Clinical } \\
\text { Trial }\end{array}$ & $\begin{array}{l}\text { Verificar os efeitos } \\
\text { do exercício WBV e } \\
\text { Pilates na DMO de } \\
\text { mulheres na pós- } \\
\text { menopausa. }\end{array}$ & $\begin{array}{l}\text { Plataformas } \\
\text { vibratórias. } \\
\text { Pilates. }\end{array}$ & $\begin{array}{c}\text { Ensaio clínico } \\
\text { randomizado e } \\
\text { controlado. }\end{array}$ & $\begin{array}{l}\text { Observou-se diferenças } \\
\text { significativas entre os grupos } \\
\text { experimentais a favor das } \\
\text { intervenções: vibração versus } \\
\text { controle e Pilates versus controle } \\
\text { para o DMO da coluna lombar e } \\
\text { trocânter. }\end{array}$ & $\begin{array}{c}\text { Três sessões semanais } \\
\text { de PV ou Pilates por } 6 \\
\text { meses proporcionaram } \\
\text { um efeito igual na } \\
\text { DMO. }\end{array}$ \\
\hline 13 & $\begin{array}{l}\text { Borba- } \\
\text { Pinheiro et } \\
\text { al. (2016) }\end{array}$ & $\begin{array}{l}\text { Resistance training } \\
\text { programs on bone } \\
\text { related variables and } \\
\text { functional } \\
\text { independence of } \\
\text { postmenopausal } \\
\text { women in } \\
\text { pharmacological } \\
\text { treatment: A } \\
\text { randomized } \\
\text { controlled trial }\end{array}$ & $\begin{array}{l}\text { Verificar os efeitos } \\
\text { de dois programas } \\
\text { lineares de } \\
\text { treinamento resistido } \\
\text { (TR) sobre a } \\
\text { densidade mineral } \\
\text { óssea (DMO), } \\
\text { autonomia funcional } \\
\text { (AF), força muscular } \\
\text { e qualidade de vida } \\
\text { (QV) de mulheres na }\end{array}$ & TR. & $\begin{array}{l}\text { Ensaio clínico } \\
\text { randomizado. }\end{array}$ & $\begin{array}{c}\text { O TR 3 / semana foi } \\
\text { significativamente mais eficiente } \\
\text { comparado a } \\
\text { TR 2 / semana, incluindo as } \\
\text { variáveis de DMO: lombar, } \\
\text { trocanter, colo fêmur e DMO total, } \\
\text { AF, força e QV } \\
\text { Além disso, os grupos TR3 e TR } 2 \\
\text { foram mais eficientes em } \\
\text { comparação com o controle para: }\end{array}$ & $\begin{array}{l}\text { Ambos os grupos } \\
\text { experimentais } \\
\text { apresentaram resultados } \\
\text { favoráveis para a DMO, } \\
\text { força, FA e QV. No } \\
\text { entanto, o TR3 } \\
\text { apresentou os melhores } \\
\text { resultados comparados } \\
\text { ao TR2 e controle }\end{array}$ \\
\hline
\end{tabular}

Persp. Online: biol \& saúde, Campos dos Goytacazes, 40 (11) 11-28, 2021

ojs3.perspectivasonline.com.br 


\begin{tabular}{|c|c|c|c|c|c|c|c|}
\hline & & & $\begin{array}{c}\text { pós-menopausa em } \\
\text { tratamento } \\
\text { farmacológico }\end{array}$ & & & $\begin{array}{c}\text { DMO total e RT2 para AF, força e } \\
\text { QV }\end{array}$ & \\
\hline 14 & $\begin{array}{l}\text { Watson et } \\
\text { al. (2018) }\end{array}$ & $\begin{array}{c}\text { High-Intensity } \\
\text { Resistance and } \\
\text { Impact } \\
\text { Training } \\
\text { Improves Bone } \\
\text { Mineral } \\
\text { Density and } \\
\text { Physical } \\
\text { Function in } \\
\text { Postmenopausa } \\
\text { I Women with } \\
\text { Osteopenia and } \\
\text { Osteoporosis: } \\
\text { The } \\
\text { LIFTMOR } \\
\text { Randomized } \\
\text { Controlled } \\
\text { Trial }\end{array}$ & $\begin{array}{l}\text { Determinar a eficácia } \\
\text { LIFTMOR e } \\
\text { monitorar os eventos } \\
\text { adversos do TR alta } \\
\text { intensidade para } \\
\text { reduzir os parâmetros } \\
\text { de risco de fratura em } \\
\text { mulheres pós- } \\
\text { menopáusicas com } \\
\text { baixa massa óssea. }\end{array}$ & TR alta intensidade & $\begin{array}{l}\text { Ensaio clínico } \\
\text { randomizado. }\end{array}$ & $\begin{array}{l}\text { Os efeitos do TR alta intensidade ( } \mathrm{n} \\
=49) \text { foram superiores }(\mathrm{p}<0,05) \text { ao } \\
\text { Controle }(\mathrm{n}=52) \text { para DMO } \\
\text { lombar, colo femoral, espessura } \\
\text { cortical, e todas as medidas de } \\
\text { autonomia funcional (AF) }\end{array}$ & $\begin{array}{l}\text { O TR alta intensidade } \\
\text { aumentou os a } \\
\text { resistência óssea, DMO } \\
\text { e AF em mulheres na } \\
\text { pós-menopausa com } \\
\text { baixa DMO. O TR alta } \\
\text { intensidade não induziu } \\
\text { eventos adversos sob } \\
\text { condições } \\
\text { supervisionadas em } \\
\text { mulheres pós- } \\
\text { menopáusicas }\end{array}$ \\
\hline
\end{tabular}


Dos 14 estudos selecionados no período de 2015 a 2020 nos bancos de dados, 9 pertenciam ao Pubmed; 4 ao BVS/MS e 1 ao Scielo. Dos nove primeiros estudos selecionados, sobre revisões sistemáticas (Quadro 1), verificou-se 12 tipos de exercícios físicos, elencados de forma isolada e combinada. Nesse sentido, os que foram evidenciados com maior frequência foram: TR $(n=5)$, Plataformas Vibratórias (PV, $n=3)$, Exercícios Aeróbicos $(n=3)$ e Exercícios com Sustentação de Peso Corporal $(n=2)$. Além disso, os exercícios com menor frequência foram: Saltos, Tai Chi, Caminhada, Exercício Aquático e Exercício em Terra..

Os resultados desta pesquisa também apresentados no Quadro 1, também mostraram cinco artigos experimentais clínicos randomizados, dos quais verificou-se 7 tipos de exercícios elencados de forma isolada e combinada: TR $(n=2),(P V, n=2)$; Aeróbicos $(n=2)$ e, com menos frequência: Drop jump, Anaeróbico, Osteoanabólicos, Pilates.

Por fim, as pesquisas foram analisadas, possibilitando a seleção, comparação e interpretação das informações coletados, resultando em um agrupamento de três categorias principais. Por meio da avaliação dos estudos, foi possível categorizar os dados sobre efeitos dos exercícios físicos na DMO, nas seguintes vertentes: A - TR isolado; B - PV; C- Exercícios multicomponentes (MC), ou seja, que envolve vários métodos de exercícios.

\section{DISCUSSÃO}

Os resultados apresentados no Quadro 1, mostraram dois estudos clínicos randomizados com método TR isolado com resultados favoráveis a melhora estatística entre outras variáveis ósseas, para DMO lombar, colo do fêmur e trocanter.

O estudo de Shojaa et al. (2020a) (Quadro 1) mostrou que as variáveis de tempo de duração da intervenção, tipo, intensidade e volume/sessão de exercícios não apresentaram diferenças significativas para coluna lombar entre os grupos, todavia, na frequência de treinamento houve efeitos estatísticos no grupo com menor frequência semanal. Na região do fêmur, as variáveis: duração da intervenção, tipo, intensidade, frequência e volume/sessão de exercícios não mostraram resultados estatísticos entre os grupos de intervenção. Ademais, as variáveis para a região do quadril total: duração, intensidade e volume/sessão de exercícios, também não evidenciaram diferença. Porém, no que se refere aos tipos e frequências de exercício, os pesos livres e frequências mais baixas demonstraram efeitos estatísticos no aumento da DMO (SHOJAA et al. 2020a).

Em contrapartida, o estudo de Borba-Pinheiro et al. (2016) apresentou dados divergentes do estudo anterior, tendo em vista que o grupo de intervenção que realizou treinos com maior frequência semanal (3 vezes/semana) obteve melhores resultados para DMO do colo do fêmur, fêmur total, trocanter, quando comparado com o grupo de menos sessões semanais e o de controle. Porém, neste mesmo estudo, o grupo com frequência semanal de duas vezes por semana teve bons resultados estatísticos comparado ao grupo controle. Dessa forma, os estudos com frequência semanal de duas vezes por semana, também podem ter efeitos positivos para DMO e variáveis de risco de quedas como a autonomia funcional foi mostrado nos estudos de Borba-Pinheiro et al. (2016) e Watson et al. (2018) apresentados no Quadro 1.

Os resultados referentes ao método de exercício com PV, que estão associados a outros exercícios, mostram que este tipo de intervenção ganhou espaço na comunidade científica por apresentar melhoras para a DMO e outras variáveis de risco de quedas. O estudo de Cascales, Arias e Alcaraz (2019) mostrou aumento para a DMO lombar e o de Oliveira et al. (2019) mostrou efeitos estatísticos para a lombar e trocanter (Quadro 1). 
O que é corroborado pelos outros estudos sobre PV, revelando particularidades para este tipo de intervenção devido ao tipo de estímulo. Na pesquisa de Mainer, Morales e Sagarra (2019), concluiu-se que as intervenções com PV aumentaram a DMO da região do colo femoral enquanto que no grupo controle diminuiu. Foi verificado também, que nas mulheres com osteoporose, a intervenção com PV resultou em um aumento na DMO da coluna lombar e do colo femoral.

Em conformidade a essas informações, o estudo de Fratini, Bonci e Bull (2016), observou que magnitudes maiores $3 \mathrm{~g}$, frequências mais baixas (de $12,5 \mathrm{~Hz}$ a $20 \mathrm{~Hz}$ ) e aparelhos de PV que alternam os lados, promoveram significativos aumentos na DMO. Com relação ao tempo de intervenção, não foi percebido diferenças significativas entre os grupos de intervenção, além disso, no que se refere a postura durante as sessões, verificou-se melhores resultados, especialmente na região do quadril e coluna lombar, quando os participantes mantem uma postura estática e em pé.

Complementando tais achados, a pesquisa de Dionello et al. (2016) demonstrou após sessões de exercícios com PV, que os biomarcadores relacionados ao metabolismo do tecido ósseo e muscular apresentaram melhorias tanto na DMO, quanto em outros parâmetros importantes como funcionais e força muscular, influenciando até mesmo na redução de quedas. Relacionado a isso, os resultados encontrados nos estudos de Cascales, Arias e Alcaraz (2019) e de Oliveira et al. (2019), mostraram que o método de PV pode ser recomendado como um tipo de exercício benefício para o aumento e controle da DMO em mulheres.

Os resultados da presente pesquisa, também evidenciaram estudos com exercícios MC, que são uma combinação de múltiplos métodos de exercícios em um programa de treinamento. Nesse sentido, a pesquisa de Cascales, Arias e Alcaraz (2019) mostrou que a combinação entre exercícios resulta em aumentos estatísticos da DMO de mulheres quando comparado o grupo controle. O que é corroborado por vários estudos apresentados no Quadro 1, com efeitos de diferentes tipos de exercícios na DMO em mulheres pós-menopáusicas utilizados como intervenção. O TR, exercício de sustentação de peso e a combinação de ambos, evidenciaram positivos aumentos da DMO da coluna lombar, colo femoral e quadril total (KEMMLER et al., 2020).

Outra pesquisa apresentada no Quadro 1, mostrou que exercícios combinados, como saltos associados ao TR e sustentação de peso do corpo, demonstraram melhores efeitos no aumento da DMO para a coluna lombar. Já exercícios sem sustentação de peso, intercalados com TR e Tai Chi, mostraram efeitos positivos no fêmur proximal. Os saltos em conjunto com TR e sustentação de peso, mostraram efeitos melhores para o quadril total (SHOJAA et al., 2020b). Para Kemmler et al. (2020) os exercícios combinados podem ser efetivos para a DMO, entretanto, para estes autores os resultados não evidenciaram diferenças significativas entre os métodos de exercícios avaliados para a DMO do fêmur e coluna lombar.

Ademais, Kim, Moon e Jin (2016) (Quadro 1) complementam destacando que o exercício físico combinando aeróbicos ao TR, evidenciam melhores resultados na DMO da coluna lombar, colo do fêmur e trocânter em mulheres pós-menopáusicas. Todavia, tais exercícios, realizados de forma separada, demonstram resultados incertos. Cascales, Arias e Alcaraz (2019) também mostraram que o exercício multicomponente (drop jump e atividade aeróbica) teve resultados positivos na DMO da região lombar e do colo do fêmur e semelhantes ao grupo que fez PV, onde não foi evidenciado diferenças entre os grupos.

Já para o estudo de Khan et al. (2019) no Quadro 1, que comparou os efeitos dos exercícios aeróbicos, anaeróbicos e osteoanabólicos (aeróbicos + anaeróbicos) na DMO,

Persp. Online: biol \& saúde, Campos dos Goytacazes, 40 (11) 11-28, 2021 
resultou em melhores efeitos a favor do grupo de exercícios osteoanabólicos, seguido pelo grupo de exercícios aeróbicos e por último os anaeróbicos. Verificou-se que os exercícios MC têm a capacidade de promover maiores efeitos para DMO.

Observou-se, também que a combinação de exercícios e suplementação de isoflavona na DMO em mulheres pós-menopáusicas (Quadro 1). Grupo que usou isoflavona e treinamentos físicos de forma isolada, com os grupos que utilizaram a suplementação de isoflavona combinada com exercícios físicos, não houve melhores benefícios na DMO no corpo inteiro, coluna lombar, quadril total, colo do fêmur e trocânter quando comparados aos controles, que receberam placebo, (QIU; MA; JIANG, 2020). Semelhante a essas informações, o estudo de Orsatti et al. (2013), mostrou que o TR de intensidade moderada e a suplementação de isoflavona não demonstrou resultados sobre a DMO e a remodelação óssea, de modo combinado ou isolado, em mulheres pós-menopáusicas, após nove meses de intervenção.

Outro tipo de exercício que é abordado com certa frequência na literatura são aqueles realizados em meio líquido (gravidade diminuída). O levantamento bibliográfico realizado por Simas et al. (2017) mostrado no Quadro 1, destacou que esses exercícios resultam em efeitos positivos para mudanças na DMO da coluna lombar e colo femoral quando comparado ao grupo. Porém, os exercícios aquáticos quando comparados aos exercícios de força, evidenciam melhoras na coluna lombar para o treino de força e não apresentam resultados estatísticos para os aquáticos. Logo, os exercícios aquáticos podem ter benefícios para a saúde óssea, mas não são tão efetivos quanto ao treinamento de força.

Diante disto, ficou evidenciado nos resultados desta pesquisa, onde os estudos clínicos controlados (Quadro 1) que se utilizaram do TR, PV e exercícios multicomponentes podem ser usados para controlar a baixa DMO e, além disso, melhorar variáveis de risco de quedas com consequências positivas para diminuir também o risco de fraturas, o que vem ao encontro dos estudos de revisão sistemática e metanálise também apresentados no Quadro 1.

Dessa forma, as pesquisas mostram uma relação benéfica entre o treinamento físico ao longo da vida e a manutenção da DMO, assim como um risco reduzido de fratura de quadril, úmero e vertebral em idades avançadas. Além disso, exercícios físicos de resistência para fortalecimento muscular, exercícios aeróbicos, com levantamento de peso e exercícios de alta intensidade, podem promover potenciais aumentos da DMO. Todavia, antes da prática de treinamentos mais intensos, recomenda-se uma avaliação profissional para determinar o quadro clínico e os limites do paciente (IOF, c2020; BRASIL, 2014).

Os exercícios físicos, portanto, são estratégias não farmacológicas que expressam efeitos positivos na prevenção e tratamento da baixa DMO, sendo possível observar evidências em estudos científicos clínicos controlados e revisões refinadas da literatura, que podem ser aplicados de forma isolada ou combinada. Nesse sentido, o ACMS (2014) e Internacional Osteoporosis Foundation (IOF, 2020) destacam que os exercícios físicos desempenham importantes funções na profilaxia da osteoporose, por elevar o pico de massa óssea durante o crescimento, reduzir a taxa de perda óssea com o avanço da idade, evita quedas e consequentemente fraturas. Além disso, contribui sobremaneira para manutenção da autonomia com independência funcional (CHAMOSCHINE et al., 2018; MANHÃES et al., 2018), pois entre vários aspectos, melhora a força muscular, flexibilidade e equilíbrio, o que foi corroborado pelo levantamento bibliográfico em bases de dados realizada na presente pesquisa.

Persp. Online: biol \& saúde, Campos dos Goytacazes, 40 (11) 11-28, 2021 


\section{CONCLUSÕES}

O presente estudo mostrou que exercícios físicos têm impacto positivo na DMO de indivíduos mais velhos e idosos. Foi possível verificar que programas de treinamento como TR, PV e também multicomponente demonstraram ser efetivos para o aumento da DMO. Observouse que o sexo feminino sofre maior impactado pela baixa DMO e as regiões ósseas mais evidenciadas foram: coluna lombar, colo do fêmur e quadril total, que se justificam pela maior prevalência de fraturas nestes. Cabe destacar, que em certa medida, todos os tipos de exercícios influenciaram na DMO ou outros fatores relacionados a risco de queda e fraturas.

É importante ainda, considerar que métodos como a hidroginástica se mostra uma escolha viável, pois mesmo que não seja o melhor exercício para DMO, há evidências de melhorara para o equilíbrio, força de membros inferiores/superiores e autonomia funcional, auxiliando assim na diminuição do risco de quedas e consequentemente, fraturas.

No público feminino, os estudos, apesar de verificarem diversos métodos de treinamento, se mostraram inconclusivos em definir um protocolo de treinamento mais eficiente para o melhoramento da DMO, devido ao uso de diferentes metodologias de intervenção. Diante, disso, ainda são necessários mais estudos, para que se possa chegar a um consenso que mostre o método de exercício mais eficiente para a DMO.

\section{REFERÊNCIAS}

ACSM. American College of Sports Medicine. Diretrizes do ACSM para os testes de esforço e sua prescrição. 9. ed. Rio de Janeiro: Guanabara, 2014.

ALMEIDA, M. M. et al. Causas e consequências de quedas de idosos atendidos em hospital público. R. Interd., v. 12, n. 1, p. 15-22, 2019. Disponível em: https://dialnet.unirioja.es/descarga/articulo/6966617.pdf Acesso em: 08 dez. 2020.

BARROS, L. C.; BELMONTE, T. S. A. As Mudanças Corporais na Envelhescência: queremos nos conscientizar disso? In: BORBA-PINHEIRO, C. J. et al. (org.). Ações multiprofissionais sobre o idoso com osteoporose: um enfoque no exercício físico. São Paulo: Yendis, 2016.

BORBA-PINHEIRO, C. J. et al. Resistance training programs on bone related variables and functional independence of postmenopausal women in pharmacological treatment: A randomized controlled trial. Archives of Gerontology and Geriatrics, v. 65, p. 36-44, 2016. Disponível em: https://pubmed.ncbi.nlm.nih.gov/26956618/ Acesso em: 18 jan. 2021.

BRASIL. Ministério da Saúde. Fundo de População da ONU alerta para violência contra idosos no Brasil. Brasília-DF, 19 jun. 2018. Disponível em: http://www.blog.saude.gov.br/index.php/promocao-da-saude/53390-fundo-de-populacao-daonu-alerta-para-violencia-contra-idosos-nobrasil\#: :text=Fundo $\% 20 \mathrm{de} \% 20$ Popula $\% \mathrm{C} 3 \% \mathrm{~A} 7 \% \mathrm{C} 3 \% \mathrm{~A} 3 \mathrm{o} \% 20 \mathrm{da} \% 20 \mathrm{ONU} \% 20$ alerta $\% 20 \mathrm{p}$ ara\%20viol\%C3\%AAncia\%20contra\%20idosos\%20no\%20Brasil,-Em\%202017\%2C\%20o\&text=Com\%20o\%20envelhecimento\%20da\%20popula\%C3\%A7\%C 3\%A3o,idade\%20contra\%20viola\%C3\%A7\%C3\%B5es\%20de\%20direitos Acesso em: 19 set. 2020. 
Ministério da Saúde. Secretaria de Atenção à Saúde. PORTARIA N 224, DE 26 DE MARÇO DE 2014. Brasília: Editora do Ministério da Saúde, 2014. Disponível em: https://portalarquivos.saude.gov.br/images/pdf/2014/abril/02/pcdt-osteoporose-2014.pdf Acesso em: 24 fev. 2021.

CÂMARA, L. C.; BASTOS, C. C.; VOLPE, E. F. T. Exercício resistido em idosos frágeis: uma revisão da literatura. Fisioter. mov., v. 25, n. 2, 2012. Disponível em: https://www.scielo.br/scielo.php?script=sci_arttext\&pid=S010351502012000200021\&lng=en \&nrm=iso Acesso em: 06 nov. 2020.

CASCALES, E. M.; ARIAS, J. A. R.; ALCARAZ, P. E. Effects of Two Different Neuromuscular Training Protocols on Regional Bone Mass in Postmenopausal Women: A Randomized Controlled Trial. Front Fhysiol., v. 10, 2019. Disponível em: https://www.ncbi.nlm.nih.gov/pmc/articles/PMC6635564/ Acesso em: 16 mar. 2021.

CHAMOSCHINE, E. M. F.; DO AMARAL, M. M.; SILVA, L. G.; BARCELOS, E. V.; CALOMENI, M. R. A influência do nível de atividade física no desempenho cognitivo e autonomia funcional de idosos. Biológicas \& Saúde, v. 8, n. 27, 14 nov. 2018. Disponível em: https://ojs3.perspectivasonline.com.br/biologicas_e_saude/article/view/1414 Acesso em: 24 mar. 2021

DIONELLO, C. F. et al. Effects of whole body vibration exercises on bone mineral density of women with postmenopausal osteoporosis without medications: novel findings and literature review. J Musculoskelet Neuronal Interact, v. 16, n. 3, p. 193-203, 2016. Disponível em: https://www.ncbi.nlm.nih.gov/pmc/articles/PMC5114342/ Acesso em: 27 jan. 2021.

DISSAT, C. Prevenção da Osteoporose: A Dose Ideal. Sociedade Brasileira de Endocrinologia e Metabologia, 31 jul. 2009. Disponível em: https://www.endocrino.org.br/prevencao-da-osteoporose-dose-ideal/ Acesso em: 09 nov. 2020.

FRATINI, A.; BONCI, T.; BULL, A. M. J. Whole Body Vibration Treatments in Postmenopausal Women Can Improve Bone Mineral Density: Results of a Stimulus Focussed Meta-Analysis. PLoS One, v. 11, n. 12, e0166774, 2016. Disponível em: https://pubmed.ncbi.nlm.nih.gov/27907000/\#: :text=Whole\%20body\%20vibration\%20was\% 20shown,exercise\%20training\%20or\%20to\%20placebo. Acesso em: 21 jan. 2021.

GALVÃO, T. F.; PANSANI, T. S. A.; HARRAD, D. Principais itens para relatar Revisões sistemáticas e Meta-análises: A recomendação PRISMA. Epidemiol. Serv. Saúde, v. 24, n. 2, 2015. Disponível em: https://www.scielo.br/scielo.php?script=sci_arttext\&pid=S223796222015000200335 Acesso em: 09 nov. 2020.

GRUPO ANIMA EDUCAÇÃO. Manual Revisão Bibliográfica Sistemática Integrativa: a pesquisa baseada em evidências. Belo Horizonte: Grupo Anima Educação, 2014. Disponível em: http://biblioteca.cofen.gov.br/wp-content/uploads/2019/06/manual_revisao_bibliograficasistematica-integrativa.pdf Acesso em: 29 dez. 2020.

INTERNACIONAL OSTEOPOROSIS FOUNDATION (IOF). Prevention-Exercise. Nyon Switzerland, c2020. Disponível em: https://www.osteoporosis.foundation/healthprofessionals/prevention/exercise Acesso em: 24 fev. 2021.

Persp. Online: biol \& saúde, Campos dos Goytacazes, 40 (11) 11-28, 2021

ojs3.perspectivasonline.com.br 
JESUS, A. P. S. et al. Efeitos do treinamento resistido sobre a osteopenia e osteoporose em idosos. Rev. Aten. Saúde, v. 17, n. 59, p. 103-110, jan./mar. 2019. Disponível em: https://seer.uscs.edu.br/index.php/revista_ciencias_saude/article/view/5007 Acesso em: 15 nov. 2020.

KEMMLER, W. et al. Effects of Different Types of Exercise on Bone Mineral Density in Postmenopausal Women: A Systematic Review and Meta-analysis. Calcif Tissue Int, n. 107, p. 409-439, 2020. Disponível em: https://link.springer.com/article/10.1007/s00223-02000744-w\#citeas Acesso em: 15 jan. 2021.

KHAN, A. A. et al. Effects of osteoanabolic exercises on bone mineral density of osteoporotic females: A randomized controlled trial. Int J Health Sci., v. 13, n. 1, p. 9-13, jan./fev. 2019. Disponível em: https://www.ncbi.nlm.nih.gov/pmc/articles/PMC6392486/ Acesso em: 16 mar. 2021.

KIM, J. E.; MOON, H.; JIN, H. M. The effects of exercise training and type of exercise training on changes in bone mineral denstiy in Korean postmenopausal women: a systematic review. J Exerc Nutrition Biochem., v. 20, n. 3, p. 7-15, 2016. Disponível em: https://www.ncbi.nlm.nih.gov/pmc/articles/PMC7325605/ Acesso em: 21 jan. 2021.

LEITE, C. R. M.; ROSA, S. S. R. F. (Org.). Novas tecnologias aplicadas à saúde: integração de áreas transformando a sociedade. Mossoró - RN: EDUERN, 2017.

MACNEE, W.; RABINOVICH, R. A.; CHOUDHURY, G. Ageing and the border between health and disease. European Respiratory Journal, Edinburgh, v. 44, n. 5, p. 1332-1352, out. 2014. Doi: 10.1183/09031936.00134014

MAINER, E. P.; MORALES, S. C.; SAGARRA, L. Efectos de las plataformas vibratorias en la salud ósea en mujeres posmenopáusicas. Rev Cubana Obstet Ginecol, v. 45, n. 1, 2019. Disponível em: http://scielo.sld.cu/scielo.php?script=sci_arttext\&pid=S0138600X2019000100118 Acesso em: 15 jan. 2021.

MANHÃES, C. B.; MARTINS, B.; BERTO, B.; NUNES, M.; SOUZA, V.; DE CAMPOS, L. P. F.; SILVA, A. T. M. F.; SANTOS, C. M. Avaliação da autonomia funcional e risco de depressão em idosos na região urbana do município de campos dos goytacazes rj. Biológicas \& Saúde, v. 8, n. 27, 14 nov. 2018. Disponível em: https://ojs3.perspectivasonline.com.br/biologicas_e_saude/article/view/1432 Acesso em: 15 jan. 2021

MENDES, K. D. S.; SILVEIRA, R. C. C. P.; GALVÃO, C. M. G. Revisão integrativa: método de pesquisa para a incorporação de evidências na saúde e na enfermagem. Texto contexto enferm., v. 17, n. 4, p. 758-64, 2008. Disponível em: https://www.scielo.br/pdf/tce/v17n4/18.pdf Acesso em: 26 set. 2020.

OLIVEIRA, L. C.; OLIVEIRA, R. G.; OLIVEIRA, D. A. A. P. Effects of Whole-Body Vibration Versus Pilates Exercise on Bone Mineral Density in Postmenopausal Women: A Randomized and Controlled Clinical Trial. J Geriatr Phys Ther., v. 42, n. 2, p. E23-E31, 2019. Disponível em: https://pubmed.ncbi.nlm.nih.gov/29443867/ Acesso em: 16 mar. 2021.

ORGANIZAÇÃO PAN-AMERICANA DE SAÚDE (OPAS). Organização Mundial da Saúde. Folha informativa - Envelhecimento e saúde. Brasília-DF, fev. 2018. Disponível em: 
https://www.paho.org/bra/index.php?option=com_content $\&$ view=article\&id=5661:folhainformativa-envelhecimento-e-saude \&Itemid=820 Acesso em: 05 nov. 2020.

ORSATTI, F. L. et al. Efeito do treinamento contrarresistência e isoflavona na densidade mineral óssea em mulheres na pós-menopausa. Rev Bras Cineantropom Desempenho Hum, v. 15, n. 6, p. 726-736, 2013. Disponível em: https://www.scielo.br/pdf/rbcdh/v15n6/a10v15n6.pdf Acesso em: 24 jan. 2021.

QIU, S.; MA, Y.; JIANG, C. Isoflavone combined with exercise on bone mineral density in postmenopausal women: A systematic review and meta-analysis of randomized controlled trials. Journal of the Chinese Medical Association, v. 83, ed. 7, p. 678-685, 2020. Disponível em:

https://journals.lww.com/jcma/Fulltext/2020/07000/Isoflavone_combined_with_exercise_on_ bone_mineral.15.aspx Acesso em: 22 jan. 2021.

SANTOS, M. L.; BORGES, G. F. Exercício físico no tratamento e prevenção de idosos com osteoporose: uma revisão sistemática. Fisioter. mov. (Impr.), Curitiba, v. 23, n. 2, p. 289-299, 2010.

Disponível: http://www.scielo.br/scielo.php?script=sci_arttext\&pid=S010351502010000200012\&lng=en \&nrm=iso Acesso em: 27 fev. 2021.

SHOJAA, M. et al. Effects of dynamic resistance exercise on bone mineral density in postmenopausal women: a systematic review and meta-analysis with special emphasis on exercise parameters. Osteoporosis, v. 31, n. 8, p. 1427-1444, maio 2020a. Disponível em: https://www.ncbi.nlm.nih.gov/pmc/articles/PMC7360540/ Acesso em: 26 jan. 2021.

SHOJAA, M. et al. Effect of Exercise Training on Bone Mineral Density in Post-menopausal Women: A Systematic Review and Meta-Analysis of Intervention Studies. Front Physiol., v. 11, p. 652, 2020b. Disponível em: https:/www.ncbi.nlm.nih.gov/pmc/articles/PMC7325605/ Acesso em: 12 jan. 2021.

SIMAS, V. et al. Effects of water-based exercise on bone health of middle-aged and older adults: a systematic review and meta-analysis. Open Access J Sports Med., v. 8, p. 39-60, 2017. Disponível em: https://www.ncbi.nlm.nih.gov/pmc/articles/PMC5376183/ Acesso em: 07 jan. 2021.

VELHO, R. B. Análise da densidade mineral óssea: comparação entre idosas praticantes da modalidade de dança aeróbia e step e idosas fisicamente inativas. 2014. Trabalho de Conclusão de Curso (Bacharel em Educação Física) - Universidade Tecnológica Federal do Paraná, Curitiba, $2014 . \quad$ Disponível em: http://repositorio.utfpr.edu.br/jspui/bitstream/1/7835/2/CT_COEFI_2014_2_11.pdf Acesso em: 16 dez. 2020.

WATSON, S. L. et al. High-intensity resistance and impact training improves bone mineral density and physical function in postmenopausal women with osteopenia and osteoporosis: the LIFTMOR randomized controlled trial. Journal of Bone and Mineral Research, v. 33, n. 2, p. 211-220, 2018. PMID: 28975661. Doi: 10.1002/jbmr.3284 